\title{
On the stability of integral sets of impulsive differential systems
}

Nikolai A. Perestyuk and Olga S. Chernikova 
Mathematical Notes, Miskolc, Vol. 2., No. 1., (2001), pp. 49-60

\title{
ON THE STABILITY OF INTEGRAL SETS OF IMPULSIVE DIFFERENTIAL SYSTEMS
}

\author{
Nikolai A. Perestyuk, Olga S. Chernikova \\ Department of Mechanics and Mathematics, Kiev National Taras Shevchenko University \\ Volodimirska 64, 01033 Kiev, Ukraine \\ pmo@mechmat.univ.kiev.ua \\ [Received February 14, 2001]
}

\begin{abstract}
The concept of stability of integral sets is introduced for impulsive differential systems of a general type (with nonfixed times of impulse effect). Sufficient conditions for the stability of integral sets are established.
\end{abstract}

Mathematical Subject Classification: 34D20

Keywords: Impulsive differential system, integral set, stable integral set

\section{Preliminaries}

Consider the impulsive differential system

$$
\begin{gathered}
\frac{\mathrm{d} x}{\mathrm{~d} t}=f(t, x), \quad t \neq t_{i}(x), \\
\left.\Delta x\right|_{t=t_{i}(x)}=I_{i}(x),
\end{gathered}
$$

where $x \in R^{n}, t \in R_{+}, f(t, x), I_{i}(x), i=1,2, \ldots$ are continuous vector-functions, functions $t_{i}(x)$ are continuous and such that $0<t_{1}(x)<t_{2}(x)<\cdots, x \in R^{n}$, $t_{i}(x) \rightarrow \infty$ as $i \rightarrow \infty$ uniformly on $x \in R^{n}$. We assume that for any point $\left(t_{0}, x_{0}\right) \in$ $R_{+} \times R^{n}$ there exists unique solution $x(t)=x\left(t, t_{0}, x_{0}\right)$ of $(1.1)$ on $\left[t_{0}, \infty\right)$ satisfying $x\left(t_{0}\right)=x_{0}$, and that the integral curve of each solution meets each of the hypersurfaces $t=t_{i}(x)$ only once, i.e., the phenomenon of "beating" is not observed. Sufficient conditions for the absence of the phenomenon of "beating" are pointed out in [1,2].

We shall recall $[1,2]$ that any solution $x\left(t, t_{0}, x_{0}\right)$ of $(1.1)$ is a piecewise continuous function with points of discontinuity

$$
\begin{aligned}
\theta_{i}^{x}, \theta_{i}^{x} & =t_{i}\left(x\left(\theta_{i}^{x}\right)\right) \quad i=1,2, \ldots ; \\
x\left(\theta_{i}^{x}, t_{0}, x_{0}\right) & =x\left(\theta_{i}^{x}-0, t_{0}, x_{0}\right), \\
x\left(\theta_{i}^{x}+0, t_{0}, x_{0}\right) & =x\left(\theta_{i}^{x}, t_{0}, x_{0}\right)+I_{i}\left(x\left(\theta_{i}^{x}, t_{0}, x_{0}\right)\right) .
\end{aligned}
$$

We shall say that set $M \subset R_{+} \times R^{n}$ is an integral set of the system (1.1), if for any point $\left(t_{0}, x_{0}\right) \in M$ it follows that $\left(t, x\left(t, t_{0}, x_{0}\right)\right) \in M$ for $t \geq t_{0}$. Note that together with point $\left(t_{0}, x_{0}\right) \in M$ belonging to the surface of discontinuity $\left(t_{0}=t_{i}\left(x_{0}\right)\right)$ the set $M$ contains also point $\left(t_{0}, x_{0}+I_{i}\left(x_{0}\right)\right)$. 
For each $i=1,2, \ldots$ denote by $\Lambda_{i}$ the set of time moments when the integral curves belonging to $M$ meet hypersurface $t=t_{i}(x)$, i.e., the set of solutions of equation $\tau=t_{i}(x(\tau)),(\tau, x(\tau)) \in M$. If, for instance, the integral set is the integral curve $\left\{(t, x): t \in R_{+}, x=x\left(t, 0, x_{0}\right)\right\}$, then $\Lambda_{i}$ consists of one point: $\Lambda_{i}=\left\{\theta_{i}^{x}\right\}$. In the case when $t_{i}(x)=\tau_{i}, i=1,2, \ldots$, each set $\Lambda_{i}$ also consists of one element : $\Lambda_{i}=\left\{\tau_{i}\right\}$.

Let $M(t)=\left\{x \in R^{n}:(t, x) \in M\right\}$. If $t=\theta_{i} \in \Lambda_{i},\left(\theta_{i}, x\right) \in M$ and $\theta_{i}=t_{i}(x)$, then, as it was noted above, the set $M$ contains both points: $\left(\theta_{i}, x\right)$ and $\left(\theta_{i}, x+I_{i}(x)\right)$, therefore, the section $M\left(\theta_{i}\right)$ contains both points $x$ and $\left.x+I_{i}(x)\right)$.

For $\theta_{i} \in \Lambda_{i}$ we make a difference between the two subsets of the section: $M_{i}\left(\theta_{i}\right)$ and $M_{i+1}\left(\theta_{i}\right)$. The one belonging to $M_{i}\left(\theta_{i}\right)$ or $M_{i+1}\left(\theta_{i}\right)$ for point $x \in R^{n}$ will be defined the following way. Let $\Omega_{i}=\left\{(t, x) \in R_{+} \times R^{n}: t_{i-1}(x)<t \leq t_{i}(x)\right\}, i=1,2, \ldots$, $t_{0}(x) \equiv 0$. If $\left(\theta_{i}, x\right) \in M \cap \Omega_{i}$, then $x \in M_{i}\left(\theta_{i}\right)$; if $\left(\theta_{i}, x\right) \in M \cap \Omega_{i+1}$ or $\left(\theta_{i}, x\right)$ is a boundary point for $M \cap \Omega_{i+1}$, then $x \in M_{i+1}\left(\theta_{i}\right) ; M\left(\theta_{i}\right)=M_{i}\left(\theta_{i}\right) \cup M_{i+1}\left(\theta_{i}\right)$ $\left(\theta_{i} \in \Lambda_{i}\right)$. It is possible that $M_{i}\left(\theta_{i}\right) \cap M_{i+1}\left(\theta_{i}\right) \neq \emptyset$.

Assume that $M(t) \neq \emptyset, M(t) \subseteq Q \subset R^{n}, t \in R_{+}$, where $Q$ is a compact set. Furthermore we shall suppose that $Q_{\varepsilon_{0}} \subset B_{h}$, where $Q_{\varepsilon_{0}}=\left\{x \in R^{n}: \rho(x, Q)<\varepsilon_{0}\right\}$, $B_{h}=\left\{x \in R^{n}:\|x\| \leq h\right\}$, for some $\varepsilon_{0}>0, h>0$.

In the case when an impulsive system (1.1) is a system with impulses at fixed times, i.e., $t_{i}(x)=\tau_{i}, i=1,2, \ldots$, we make a difference between the two subsets of the section of integral set $M$ only for points $\tau_{i}$ : if $\left(\tau_{i}, x\right) \in M \cap \Omega_{i}$, then $x \in M_{i}\left(\tau_{i}\right)$; if $\left(\tau_{i}, x\right) \in M$ and $\left(\tau_{i}, x\right)$ is a boundary point of $\Omega_{i+1} \cap M$, then $x \in M_{i+1}\left(\tau_{i}\right)$.

We shall say that the solution $y(t)$ of (1.1) belongs to $\varepsilon$ - neighbourhood of the integral set $M$ for $t \geq t_{0}$ if there exists a sequence $\left\{\theta_{i}\right\}, i=1,2, \cdots, \theta_{i} \in \Lambda_{i}$, such that $\left|\theta_{i}^{y}-\theta_{i}\right|<\varepsilon$ and $\rho\left(y\left(t, t_{0}, y_{0}\right), M(t)\right)<\varepsilon$ for $t \geq t_{0}, t \notin \bigcup_{i=1}^{\infty}\left(\theta_{i}-\varepsilon, \theta_{i}+\varepsilon\right.$ ) (here $\theta_{i}^{y}, i=1,2, \ldots$, are points of discontinuity of the solution $y(t)$ and $\rho(y, M(t))$ is a distance between $y$ and the set $M(t)$ ).

With every point $\left(t_{0}, y_{0}\right) \in R_{+} \times R^{n}$ we connect a set $M_{y_{0}}\left(t_{0}\right)$ as follows:

$$
M_{y_{0}}\left(t_{0}\right)=\left\{\begin{array}{lll}
M\left(t_{o}\right), & \text { if } \quad t_{0} \notin \bigcup_{i=1}^{\infty} \Lambda_{i}, \\
M_{i}\left(t_{o}\right), & \text { if } \quad t_{0} \in \Lambda_{i},\left(t_{0}, y_{0}\right) \in \Omega_{i}, \\
M_{i+1}\left(t_{o}\right), & \text { if } \quad t_{0} \in \Lambda_{i},\left(t_{0}, y_{0}\right) \in \Omega_{i+1} .
\end{array}\right.
$$

We shall give the following definitions of stability and asymptotic stability of an integral set.

The integral set $M$ of system (1.1) is stable if for any $\varepsilon>0$ and $t_{0} \in R_{+}$there exists such a number $\delta=\delta\left(\varepsilon, t_{0}\right)>0$ that for any solution $y(t)=y\left(t, t_{0}, y_{0}\right)$ of system (1.1),$\rho\left(y_{0}, M_{y_{0}}\left(t_{0}\right)\right)<\delta$ implies that $y\left(t, t_{0}, y_{0}\right)$ belongs to $\varepsilon$ - neighbourhood of the integral set $M$ for $t \geq t_{0}$ (it is assumed that $t_{0} \neq \theta_{i}^{y}$ ).

The stable integral set $M$ of system (1.1) is asymptotically stable if there exists such a number $\delta_{0}>0$ that for any solution $y(t)=y\left(t, t_{0}, y_{0}\right)$ of (1.1) that satisfies the inequality $\rho\left(y_{0}, M_{y_{0}}\left(t_{0}\right)\right)<\delta_{0}$, the following holds: $\lim _{t \rightarrow \infty} \rho(y(t), M(t))=0$. 


\section{Reduction to a system with impulses at fixed times}

Suppose that in (1.1) functions $f(t, x)$ and $I_{i}(x)$ satisfy the conditions:

$$
\|f(t, x)-f(t, y)\|+\left\|I_{i}(x)-I_{i}(y)\right\| \leq K\|x-y\|
$$

uniformly with respect to $t \in R_{+}, I=1,2, \ldots$ for all $x, y \in R^{n}$, and

$$
\sup _{t \geq 0}\|f(t, 0)\|+\sup _{t \geq 1}\left\|I_{i}(0)\right\|=N<+\infty \text {. }
$$

Suppose that for $x \in B_{h}$ the equations of surfaces of discontinuity of solutions can be written in the form:

$$
t=t_{i}(x)=\tau_{i}+\tau_{i}(x), \quad i=1,2, \ldots,
$$

and the following conditions are satisfied

$$
\begin{aligned}
& \tau_{i+1}-\tau_{i} \geq d>0, \quad 0 \leq \tau_{i}(x) \leq l<d, \quad i=1,2, \ldots \\
& \left|\tau_{i}(x)-\tau_{i}(y)\right| \leq l\|x-y\|
\end{aligned}
$$

where $l$ is a sufficiently small number.

Under such assumptions investigation of the stability of an integral set of impulsive differential system (1.1) can be reduced to an investigation of the stability of an integral set of some differential system with impulses at fixed times.

Let $M$ be an integral set of the system (1.1). Consider set $\hat{M}$ which differs from set $M$ in points $(t, x)$ disposed between surfaces $t=\tau_{i}$ and $t=t_{i}(x), i=1,2, \ldots$, as follows. Each point $\left(t^{*}, x^{*}\right) \in M$, such that $\tau_{i}<t^{*} \leq t_{i}\left(x^{*}\right)$, is replaced by point $\left(t^{*}, \tilde{x}\left(t^{*}, \theta_{i}^{x}, x\left(\theta_{i}^{x}+0\right)\right)\right.$, where $\tilde{x}(t)$ is a solution of system $\frac{d \tilde{x}}{d t}=f(t, \tilde{x})$ with initial condition $\tilde{x}\left(\theta_{i}^{x}\right)=x\left(\theta_{i}^{x}+0\right)=x\left(\theta_{i}^{x}+0, t^{*}, x^{*}\right)\left(\theta_{i}^{x}\right.$ denotes the moment at which the integral curve $\left\{t, x\left(t, t^{*}, x^{*}\right)\right\}, t \geq t^{*}$, of (1.1) meets a surface $\left.t=t_{i}(x)\right)$.

Let $x(t)$ be a solution of (1.1) with initial condition $x\left(\tau_{i}\right)=x$. Consider function

$$
G_{i}(x)=\int_{\theta_{i}^{x}}^{\tau_{i}} f(\tau, \tilde{x}(\tau)) \mathrm{d} \tau+I_{i}\left(x\left(\theta_{i}^{x}\right)\right)+\int_{\tau_{i}}^{\theta_{i}^{x}} f(\tau, x(\tau)) \mathrm{d} \tau .
$$

Proceeding from the construction of set $\hat{M}$ one can easily make sure that the following statement is true.

Lemma 1. If $M$ is an integral set of system (1.1), then $\hat{M}$ is an integral set of the differential system with impulses at fixed times

$$
\begin{aligned}
& \frac{\mathrm{d} x}{\mathrm{~d} t}=f(t, x), \quad t \neq \tau_{i} \\
& \left.\quad \Delta x\right|_{t=\tau_{i}}=G_{i}(x)
\end{aligned}
$$

Applying the Gronwall-Bellman inequality and taking into consideration conditions (2.1)-(2.4) we set the following statement . 
Lemma 2. Let $x \in B_{h}$. Then the solution $x(t)$ satisfies the inequality

$$
\|x(t)\| \leq H, t \in\left[\tau_{i}, \tau_{i}+l\right]
$$

where $H=\left\{(1+K)(h+N l) e^{K l}+N(1+l)\right\} e^{K l}$.

Lemma 3. [3]. Let $\theta_{i}^{x}$ and $\theta_{i}^{y}$ be the points of discontinuity of solutions $x(t)$ and $y(t)$ of (1.1) with initial conditions $x\left(\tau_{i}\right)=x, y\left(\tau_{i}\right)=y\left(x, y \in B_{h}\right)$. Then

$$
\left|\theta_{i}^{x}-\theta_{i}^{y}\right| \leq \frac{l e^{K l}}{1-l(N+K H)}\|x-y\|
$$

$\left(l<(N+K H)^{-1}\right)$.

Theorem 1. The integral set $M$ of the differential impulsive system (1.1) is stable if the integral set $\hat{M}$ of differential system (2.5) with impulses at fixed times is stable.

Proof. Let integral set $\hat{M}$ of system (2.5) be stable. Choose and fix $t_{0} \in R^{+}$.

Consider at first a case when $t_{0} \notin \bigcup_{i=1}^{\infty}\left[\tau_{i}, T_{i}\right)$, where $T_{i}=\sup \Lambda_{i}, i=1,2, \ldots$ According to the definition of the stability of an integral set for a differential system with impulses at fixed times, for arbitrary number $\varepsilon>0\left(\varepsilon<\varepsilon_{0}\right)$ there exists such a number $\delta>0$ that for any solution $\hat{y}\left(t, t_{0}, y_{0}\right)$ of $(2.5)$ inequality $\rho\left(y_{0}, \hat{M}\left(t_{0}\right)\right)<\delta$ implies

$$
\rho\left(\hat{y}\left(t, t_{0}, y_{0}\right), \hat{M}(t)\right)<\varepsilon
$$

for $t \geq t_{0}\left(t \neq \tau_{i}\right)$ and $\rho\left(\hat{y}\left(\tau_{i}, t_{0}, y_{0}\right), \hat{M}_{i}\left(\tau_{i}\right)\right)<\epsilon\left(\tau_{I} \geq t_{0}\right)$. The last means that for each $i$ such that $\tau_{i} \geq t_{0}$, there exists $x_{i} \in \hat{M}_{i}\left(\tau_{i}\right)$ which satisfies inequality $\left\|\hat{y}\left(\tau_{i}, t_{0}, y_{0}\right)-x_{i}\right\|<\varepsilon$. Because solution $\hat{y}(t)=y\left(\tau_{i}, t_{0}, y_{0}\right)$ of system (2.5) coincides with solution $y(t)=y\left(t, t_{0}, y_{0}\right)$ of system (1.1) in all of points except points from $\bigcup_{i: \tau_{i} \geq t_{0}}\left(\tau_{i}, \theta_{i}^{y}\right)$ and sets $M$ and $\hat{M}$ differ from one another only for $t \in \bigcup_{i=1}^{\infty}\left(\tau_{i}, T_{i}\right)$, where $T_{i}=\sup \Lambda_{i}, \quad i=1,2, \ldots$, then $\rho(y(t), M(t))<\varepsilon$ for $t \notin \underset{i: \tau_{i} \geq t_{0}}{\bigcup}\left(\tau_{i}, \gamma_{i}\right)$, $\gamma_{i}=\max \left(\theta_{i}^{y}, T_{i}\right)$. Estimate distance $\rho(y(t), M(t))$ for $t \in\left(\tau_{i}, \gamma_{i}\right)$, supposing, for example, that $\gamma_{i}=T_{i}$. Let $x(t), t \geq \tau_{i}$, be a solution of system (1.1) with initial condition $x\left(\tau_{i}\right)=x_{i}$. In obedience to lemma 3 for time moments $\theta_{i}^{x}, \theta_{i}^{y}\left(\theta_{i}^{x} \in \Lambda_{i}\right)$ we have the following

$$
\left|\theta_{i}^{x}-\theta_{i}^{y}\right| \leq \frac{l e^{K L}}{1-l(N+K H)} \varepsilon
$$

Without loss of generality, we may suppose that $\theta_{i}^{y}<\theta_{i}^{x}$. It is easy to verify that for $\tau_{i}<t \leq \theta_{i}^{y}$ the following inequality is fulfilled

$$
\|y(t)-x(t)\| \leq\left\|y\left(\tau_{i}\right)-x\left(\tau_{i}\right)\right\| e^{K\left(t-\tau_{i}\right)}<\varepsilon e^{K\left(\theta_{i}^{y}-\tau_{i}\right)}
$$

and also for $\theta_{i}^{x}<t \leq T_{i}$ - inequality

$$
\|y(t)-x(t)\| \leq\left[(1+K) \epsilon e^{K l}+(2+K)(K H+N)\left(\theta_{i}^{x}-\theta_{i}^{y}\right)\right] e^{K\left(i-\theta_{i}^{x}\right)}
$$


Let $\varepsilon_{1}$ be an arbitrary sufficiently small number $\left(\varepsilon_{1}, \varepsilon_{0}\right)$ and $\varepsilon<\frac{\varepsilon_{1}}{A}$, where

$$
A=\max \left\{e^{2 K l}\left[1+K+\frac{(2+K)(N+K H) l}{1-l(N+K H)}\right], \frac{l e^{K l}}{1-l(N+K H)}\right\} .
$$

From the stability of integral set $\hat{M}$ for system (2.5) and inequalities (2.6) - (2.9) it follows that for any solution $y\left(t, t_{0}, y_{0}\right)$ of system $(1.1)$, which satisfies inequality $\rho\left(y_{0}, M\left(t_{0}\right)\right)<\delta$, inequality $\rho\left(y\left(t, t_{0}, y_{0}\right), M(t)\right)<\varepsilon_{1}$ holds for

$$
t \geq t_{0}, t \notin \bigcup_{i=1}^{\infty}\left(\theta_{i}-\varepsilon_{1}, \theta_{i}+\varepsilon_{1}\right)
$$

where $\theta_{i}$ is some point of set $\Lambda_{i}, i=1,2, \ldots$, i.e., it follows that integral set $M$ of system (1.1) is stable.

It is not difficult to show that if $t_{0} \in \bigcup_{i=1}^{\infty}\left[\tau_{i}, T_{i}\right)$, then from $\rho\left(y_{0}, M_{y_{0}}\left(t_{0}\right)\right)<\delta_{1}$ where $\delta_{1}<\frac{\delta}{A}$, follows the inequality $\rho\left(y\left(i, i_{0}, y_{0}\right), M(t)\right)<\delta$ on $\left[i_{0}, \tau_{i}+l\right]$, except, perhaps, of points of some interval $\left(\alpha_{i}^{y_{0}}, \beta_{i}^{y_{0}}\right)$, the length of which does not exceed $\delta$. Taking into account this fact and the stability of the set $\hat{M}$ for system (2.5), we conclude that the integral set $M$ of system (1.1) is stable.

The next assertion is also true:

Theorem 2. The integral set $M$ of differential impulsive system (1.1) is asymptotically stable if the integral set $\hat{M}$ of differential system (2.5) with impulses at fixed times is asymptotically stable.

\section{Stability of the integral set for a system with impulses at fixed times}

We shall give sufficient conditions for the stability (asymptotic stability) of an integral set of an impulsive differential system with fixed time moments sequence of impulse action

$$
\begin{aligned}
& \frac{\mathrm{d} x}{\mathrm{~d} t}=F(t, x), \quad t \neq \tau_{i}, \\
& \left.\Delta x\right|_{t=t_{i}}=J_{i}(x),
\end{aligned}
$$

where $x \in R^{n}, t \in R_{+}, i=1,2, . ., 0=\tau_{0}<\tau_{1}<\tau_{2}<\ldots, \quad \tau_{i} \rightarrow \infty$ as $i \rightarrow \infty$.

For the establishment of the stability conditions of integral sets of an impulsive differential system (3.1) we will employ a method of auxiliary functions (the second Lyapunov method), which, as it is known, is effectively applicable for the study of stability of sets in the theory of usual differential equations, in particular, the stability of invariant sets of dynamic systems [4-8], and also for research of various stability questions in the theory of impulse differential equations. We will apply means analogous to those which were applied in [1, 2, 9-15].

As above, assume that system (3.1) satisfies the conditions of existence and uniqueness of solutions, and that any solution of the system $(3.1)$ is defined on $\left[t_{0},+\infty\right)$. 
Let $M$ be an integral set of system (3.1). As above, suppose, that for each $t \in R_{+}$ section $M(t)$ is not empty and is contained in compact set $Q \subset R^{n} ; Q_{\varepsilon_{0}} \subseteq B_{h}$.

Consider continuously differentiable function $V(t, x)$ defined in domain $Z=$ $\left\{(t, x): t \in R_{+}, x \in Q_{\varepsilon_{0}}\right\}$, which satisfies such properties:

$$
\begin{gathered}
V(t, x)=0, \quad(t, x) \in M ; \quad V(t, x)>0,(t, x) \notin M ; \\
V(t, x) \geq a(\rho(x, M(t))),
\end{gathered}
$$

where $a(s)(s \geq 0)$ is a continuous increasing function, $a(0)=0$.

Assume that there exists such $\mu>0 \quad\left(0<\mu<\varepsilon_{0}\right)$, that for $x \in Q_{\mu}$ will be $x+J_{i}(x) \in Q_{\varepsilon_{0}}, \quad i=1,2, \ldots$

Theorem 3. If for system (3.1) there exists a continuously differentiable function $V(t, x)$ satisfying in domain $Z$ the conditions (3.2), (3.3) and conditions

$$
\begin{gathered}
\frac{\partial V(t, x)}{\partial t}+\left\langle\operatorname{grad}_{x} V(t, x), F(t, x)\right\rangle \leq 0, \\
V\left(\tau_{i}, x+J_{i}(x)\right) \leq V\left(\tau_{i}, x\right), \quad i=1,2, \ldots,
\end{gathered}
$$

then the set $M$ is stable with respect to system (3.1).

Proof. Let $t_{0} \in R_{+}$and $\varepsilon>0$ be a sufficiently small number. Taking into consideration properties of the function $V(t, x)$ we can indicate such $\delta>0$, that $V\left(t_{0}, x\right)<a(\varepsilon)$ if $\rho\left(x, M\left(t_{0}\right)\right)<\delta$. Let $\rho\left(x_{0}, M\left(t_{0}\right)\right)<\delta$ and $x(t)=x\left(t, t_{0}, x_{0}\right)$ be a solution of the system (3.1). Evidently, that function $v(t)=V(t, x(t))$ under conditions (3.4), (3.5) is unincreasing; $v(t) \leq v\left(t_{0}\right), t \geq t_{0}$. The assumption that at some time moment $t^{*}>t_{0}$ we shall have $\rho\left(x\left(t^{*}\right), M\left(t^{*}\right)\right) \geq \varepsilon$, leads to the contradictory inequalities:

$$
a(\varepsilon) \leq a\left(\rho\left(x\left(t^{*}\right), M\left(t^{*}\right)\right) \leq v\left(t^{*}\right) \leq v\left(t_{0}\right)<a(\varepsilon) .\right.
$$

This contradiction proves Theorem 3 .

Theorem 4. Assume that for system (3.1) there exists a continuously differentiable function $V(t, x)$ satisfying in domain $Z$ the conditions (3.2), (3.3) and conditions

$$
\begin{gathered}
\frac{\partial V(t, x)}{\partial t}+\left\langle\operatorname{grad}_{x} V(t, x), F(t, x)\right\rangle \leq-\alpha(t) \varphi(V(t, x)) \\
V\left(\tau_{i}, x+J_{i}(x)\right) \leq \psi_{i}\left(V\left(\tau_{i}, x\right)\right), \quad i=1,2, \ldots,
\end{gathered}
$$

where $\alpha(t),\left(t \in R_{+}\right)$is continuous nonnegative function, $\varphi(s), \psi_{i}(s)$ are continuous functions, $\varphi(s)>0, \psi_{i}(s)>0$ for $s>0$ and $\varphi(0)=\psi_{i}(0)=0$.

If there exists a positive number $a_{0}$ such, that for all $a \in\left(0, a_{0}\right]$ the inequalities

$$
\int_{a}^{\psi_{i}(a)} \frac{\mathrm{d} s}{\varphi(s)} \leq \int_{\tau_{i-1}}^{\tau_{i}} \alpha(t) \mathrm{d} t \quad i=1,2, \ldots
$$


hold, then the set $M$ is stable with respect to system (3.1).

Proof. Let $\varepsilon>0$ be an arbitrary number such that $\varepsilon<\mu, a(\varepsilon)<a_{0}$, and let $t_{0} \in\left(\tau_{j}, \tau_{j+1}\right]$. For arbitrary $\delta>0$, denote by $m_{\delta}=\sup _{\rho\left(x, M\left(t_{0}\right)\right)<\delta} V\left(t_{0}, x\right)$. Choose $\delta>0$ so small that inequalities $m_{\delta}<a(\varepsilon), \sup _{0 \leq s \leq m_{\delta}} \psi_{j+1}(s)<a(\varepsilon)$ hold.

Let $\quad x(t)=x\left(t, t_{0}, x_{0}\right)$ be an arbitrary solution of system (3.1), such that $\rho\left(x_{0}, M\left(t_{0}\right)\right)<\delta$. Prove that $\rho(x(t), M(t))<\varepsilon$ for $t \geq t_{0}$. Suppose the contrary: in some time moment $t^{*}>t_{0}$ will be $\rho\left(x\left(t^{*}\right), M\left(t^{*}\right)\right) \geq \varepsilon$. First such a time moment can be only a moment of impulse action. Assume that $\tau_{k}$ is such time moment. Evidently, $k \geq j+2$. Consider function $v(t)=V(t, x(t))$. In obedience to our supposition as for behaviour of solution $x(t)$ the next inequalities hold $v(t)<$ $a(\varepsilon), t_{0} \leq t \leq \tau_{k}$, and $v\left(\tau_{k}+0\right) \geq a(\varepsilon)$. In view of the conditions of theorem

$$
\int_{v\left(\tau_{i}\right)}^{v\left(\tau_{i}+0\right)} \frac{\mathrm{d} s}{\varphi(s)} \leq \int_{v\left(\tau_{i}\right)}^{\psi_{i}\left(v\left(\tau_{i}\right)\right)} \frac{\mathrm{d} s}{\varphi(s)} \leq \int_{\tau_{i-1}}^{\tau_{i}} \alpha(t) \mathrm{d} t, \quad i=j+2, \ldots, k
$$

and

$$
\int_{v\left(\tau_{i-1}+0\right)}^{v\left(\tau_{i}\right)} \frac{\mathrm{d} s}{\varphi(s)} \leq-\int_{\tau_{i-1}}^{\tau_{i}} \alpha(t) \mathrm{d} t \quad i=j+2, \ldots, k
$$

So,

$\int_{v\left(\tau_{j+1}+0\right)}^{v\left(\tau_{k}+0\right)} \frac{d s}{\varphi(s)}=\sum_{j+2}^{k} \int_{v\left(\tau_{i-1}+0\right)}^{v\left(\tau_{i}\right)} \frac{d s}{\varphi(s)}+\sum_{j+2}^{k} \int_{v\left(\tau_{i}\right)}^{v\left(\tau_{i}+0\right)} \frac{d s}{\varphi(s)} \leq \int_{\tau_{j+1}}^{\tau_{k}} \alpha(t) d t-\int_{\tau_{j+1}}^{\tau_{k}} \alpha(t) d t=0$ or $v\left(\tau_{k}+0\right) \leq v\left(\tau_{j+1}+0\right)$ and $v\left(\tau_{k}+0\right)<a(\varepsilon)$. The contradiction obtained proves the theorem.

Consider a case when sequence $\left\{\tau_{i}\right\}, i=1,2, \ldots$, satisfies a condition

$$
\tau_{i+1}-\tau_{i} \geq \theta, \quad \theta>0
$$

Theorem 5. Let for system (3.1) there exist a continuously differentiable function $V(t, x)$ satisfying in domain $Z$ the conditions (3.2), (3.3) and condition

$$
\begin{gathered}
\frac{\partial V(t, x)}{\partial t}+\left\langle\operatorname{grad}_{x} V(t, x), F(t, x)\right\rangle \leq-\varphi(V(t, x)) \\
V\left(\tau_{i}, x+J_{i}(x)\right) \leq \psi\left(V\left(\tau_{i}, x\right)\right), \quad i=1,2, \ldots
\end{gathered}
$$

where $\varphi(s), \psi(s)$ are continuous functions, $\varphi(s)>0, \psi(s)>0$ for $s>0 \mathrm{i}$ $\varphi(0)=\psi(0)=0$.

If sequence $\left\{\tau_{i}\right\}, i=1,2, \ldots$, satisfies condition (3.9) and there exists a positive number $a_{0}$ such that for arbitrary $a \in\left(0, a_{0}\right]$ 


$$
\int_{a}^{\psi(a)} \frac{d s}{\varphi(s)} \leq \theta,
$$

then the set $M$ is stable with respect to system (3.1).

Assertion of Theorem 5, which extends the results [10] about the stability of a trivial solution of an impulsive differential system to the case of stability of integral sets, immediately follows from Theorem 4.

Theorem 6. Let for system (3.1) there exist a continuously differentiable function $V(t, x)$ satisfying in domain $Z$ the conditions (3.2), (3.3) and conditions

$$
\begin{gathered}
\frac{\partial V(t, x)}{\partial t}+\left\langle\operatorname{grad}_{x} V(t, x), F(t, x)\right\rangle \leq-\alpha(t) \varphi(V(t, x)) \\
V\left(\tau_{i}, x+J_{i}(x)\right) \leq \psi_{i}\left(V\left(\tau_{i}, x\right)\right), \quad i=1,2, \ldots,
\end{gathered}
$$

where $\alpha(t) \quad\left(t \in R_{+}\right)$is a continuous nonnegative function, $\varphi(s), \psi_{i}(s), i=1,2, \ldots$, are continuous functions, $\varphi(s)>0, \psi_{i}(s)>0$ for $s>0$ and $\varphi(0)=\psi_{i}(0)=0$.

If there exists a positive number $a_{0}$ such that for all $a \in\left(0, a_{0}\right]$ the following inequalities

$$
\int_{\psi_{i}(a)}^{a} \frac{d s}{\varphi(s)} \geq \int_{\tau_{i}}^{\tau_{i}+1} \alpha(t) d t, \quad i=1,2, \ldots,
$$

hold, then the set $M$ is stable with respect to system (3.1).

Proof. Let $\varepsilon>0$ be an arbitrary sufficiently small number $\left(\varepsilon<\mu, a(\varepsilon)<a_{0}\right)$, and let $t_{0} \in\left(\tau_{j}, \tau_{j+1}\right], j \geq 1$. For arbitrary $\delta>0$ note $m_{\delta}=\sup _{\rho\left(x, M\left(t_{0}\right)\right)<\delta} V\left(t_{0}, x\right)$.

Choose $\delta>0$ so small that inequality $m_{\delta}<\min \left\{a(\varepsilon), \psi_{j}(a(\varepsilon))\right\}$ holds. Let $x(t)=x\left(t, t_{0}, x_{0}\right)$ be an arbitrary solution of system $(3.1)$ such that $\rho\left(x_{0}, M\left(t_{0}\right)\right)<\delta$. Prove that $\rho(x(t), M(t))<\varepsilon$ for $t \geq t_{0}$. Suppose the contrary, i.e., that there exists $t^{*}>t_{0}, t^{*} \in\left(\tau_{k}, \tau_{k+1}\right], k \geq j$, such that $\rho\left(x\left(t^{*}\right), M\left(t^{*}\right)\right) \geq \varepsilon$. Suppose at first that $k=j$ and $t^{*} \in\left(t_{0}, \tau_{j+1}\right]$. In view of (3.12) for function $v(t)=V(t, x(t))$ have:

$$
\int_{v\left(t_{0}\right)}^{v\left(t^{*}\right)} \frac{\mathrm{d} s}{\varphi(s)} \leq \int_{t_{0}}^{t^{*}} \alpha(s) \mathrm{d} s \leq \int_{\tau_{j}}^{\tau_{j+1}} \alpha(s) \mathrm{d} s .
$$

On the other hand, $v\left(t^{*}\right) \geq a(\varepsilon)$, and

$$
\int_{v\left(t_{0}\right)}^{v\left(t^{*}\right)} \frac{\mathrm{d} s}{\varphi(s)}>\int_{\psi_{j}(a(\varepsilon))}^{a(\varepsilon)} \frac{\mathrm{d} s}{\varphi(s)},
$$

so,

$$
\int_{\psi_{j}(a(\varepsilon))}^{a(\varepsilon)} \frac{\mathrm{d} s}{\varphi(s)}<\int_{\tau_{j}}^{\tau_{j+1}} \alpha(s) \mathrm{d} s
$$


that contradicts condition (3.14). Thus $v(t)<a(\varepsilon), t \in\left[t_{0}, \tau_{j+1}\right]$ (in particular, $\left.v\left(\tau_{j+1}\right)<a(\varepsilon)\right)$.

Assume now that $t^{*} \in\left(\tau_{k}, \tau_{k+1}\right], k \geq j+1, \rho(x(t), M(t))<\varepsilon$ for $t_{0} \geq t \geq \tau_{k}$, and that $v\left(\tau_{k}\right)<a(\varepsilon)$. Evidently, one can think that $\varepsilon \leq \rho\left(x\left(t^{*}\right), M\left(t^{*}\right)\right)<\varepsilon_{0}$; also $\rho(x(t), M(t))<\varepsilon_{0}$ for $t \in\left[t_{0}, t^{*}\right]$.

In view of conditions of theorem the next correlations hold for $t \in\left(\tau_{k}, \tau_{k+1}\right]$ :

$$
\begin{gathered}
\int_{v\left(\tau_{k}\right)}^{v\left(t^{*}\right)} \frac{\mathrm{d} s}{\varphi(s)}=\int_{v\left(\tau_{k}\right)}^{v\left(\tau_{k}+0\right)} \frac{\mathrm{d} s}{\varphi(s)}+\int_{v\left(\tau_{k}+0\right)}^{v\left(t^{*}\right)} \frac{\mathrm{d} s}{\varphi(s)} \leq \\
\leq \int_{v\left(\tau_{k}\right)}^{\psi_{k}\left(v\left(\tau_{k}\right)\right)} \frac{\mathrm{d} s}{\varphi(s)}+\int_{v\left(\tau_{k}+0\right)}^{v\left(t^{*}\right)} \frac{\mathrm{d} s}{\varphi(s)} \leq-\int_{\tau_{k}}^{\tau_{k+1}} \alpha(s) \mathrm{d} s+\int_{\tau_{k}}^{\tau_{k+1}} \alpha(s) \mathrm{d} s=0,
\end{gathered}
$$

so $v\left(t^{*}\right) \leq v\left(\tau_{k}\right), \quad t \in\left(\tau_{k}, \tau_{k+1}\right]$. Supposing that $\rho\left(x\left(t^{*}\right), M\left(t^{*}\right)\right) \geq \varepsilon$ we obtain a contradiction: $a(\varepsilon) \leq v\left(t^{*}\right) \leq v\left(\tau_{k}\right)<a(\varepsilon)$. Thus $\rho(x(t), M(t))<\varepsilon$ for $t \in$ $\left(\tau_{k}, \tau_{k+1}\right]$. From the reasoning above we conclude that $v(t) \leq v\left(t_{0}\right)$ on $\left[t_{0}, \infty\right)$ and also $\rho(x(t), M(t))<\varepsilon, t \geq t_{0}$. The theorem is proved.

Suppose now that the time moments of impulsive action satisfy condition

$$
\tau_{i+1}-\tau_{i} \leq \theta_{1}, \quad \theta_{1}>0, \quad i=1,2, \ldots
$$

Theorem 7. Let for system (3.1) there exists a continuously differentiable function $V(t, x)$ satisfying in domain $Z$ conditions $(3.2),(3.3)$ and conditions

$$
\begin{gathered}
\frac{\partial V(t, x)}{\partial t}+\left\langle\operatorname{grad}_{x} V(t, x), F(t, x)\right\rangle \leq \varphi(V(t, x)) \\
V\left(\tau_{i}, x+J_{i}(x)\right) \leq \psi\left(V\left(\tau_{i}, x\right)\right), \quad i=1,2, \ldots
\end{gathered}
$$

where $\varphi(s), \psi_{i}(s), i=1,2, \ldots$, are continuous functions, $\varphi(s)>0, \psi_{i}(s)>0$ for $s>0$ and $\varphi(0)=\psi_{i}(0)=0$.

If there exists a positive number $a_{0}$ such that for all $a \in\left(0, a_{0}\right]$ the following inequality

$$
\int_{\psi(a)}^{a} \frac{\mathrm{d} s}{\varphi(s)} \geq \theta_{1}
$$

holds, then the set is $M$ stable with respect to system (3.1).

Verification of the assertion of Theorem 7 evidently follows from Theorem 6.

The next assertion also follows from Theorems 4 and 6 .

Theorem 8. Let for system (3.1) there exist a continuously differentiable function $V(t, x)$ satisfying in domain $Z$ conditions $(3.2),(3.3),(3.10),(3.11)$. 
Assume that sequence $\left\{\tau_{i}\right\}, i=1,2, \ldots$ is such that for sufficiently large $t$ ( $t>$ $\left.T_{0} \geq t_{0}\right)$

$$
\frac{i\left(t, t_{0}\right)}{t-t_{0}} \leq p, \quad p=\text { const }
$$

where by $i\left(t, t_{0}\right), t \geq t_{0}$, a number of impulses are denoted on $\left[t_{0}, t\right]$.

Then if there exists such $a_{0}>0$ that for all $a \in\left(0, a_{0}\right]$

$$
\int_{a}^{\psi(a)} \frac{\mathrm{d} s}{\varphi(s)} \leq \frac{1}{p}
$$

then the set $M$ is stable with respect to system (3.1).

Theorem 9. Let for system (3.1) there exist a continuously differentiable function $V(t, x)$ satisfying in domain $Z$ conditions (3.2), (3.3), (3.16), (3.17).

Assume that sequence $\left\{\tau_{i}\right\}, i=1,2, \ldots$ is such that for sufficiently large $t$ ( $t>$ $\left.T_{0} \geq t_{0}\right)$

$$
\frac{i\left(t, t_{0}\right)}{t-t_{0}} \geq p, p>0, p=\text { const. }
$$

Then if there exists such $a_{0}>0$ that for all $a \in\left(0, a_{0}\right]$

$$
\int_{\psi(a)}^{a} \frac{\mathrm{d} s}{\varphi(s)} \geq \frac{1}{p}
$$

then the set $M$ is stable with respect to system (3.1).

We shall present the sufficient conditions for the asymptotic stability of an integral set.

Theorem 10. Let for system (3.1) there exist a continuously differentiable function $V(t, x)$ satisfying in domain $Z$ conditions (3.2), (3.3), (3.6), (3.7).

Then if there exists such $a_{0}>0$ that for all $a \in\left(0, a_{0}\right]$

$$
\int_{(a)}^{\psi_{i}(a)} \frac{\mathrm{d} s}{\varphi(s)} \leq \int_{\tau_{i-1}}^{\tau_{i}} \alpha(t) \mathrm{d} t-\gamma_{i} \quad i=1,2, \ldots
$$

where $\gamma_{i} \geq 0, \sum_{i=1}^{\infty} \gamma_{i}=\infty$, then the set $M$ is asymptotically stable with respect to system (3.1).

Proof. Since the conditions of Theorem 4 are fulfilled, then the integral set of system (3.1) is stable. So for given $t_{0}$ (let $t_{0} \in\left(\tau_{j}, \tau_{j+1}\right]$ ) and arbitrary sufficiently small $\varepsilon>0)$ there exists such $\delta>0$ that for arbitrary solution $x(t)=x\left(t, t_{0}, x_{0}\right)$ of 
(3.1) which satisfies $\rho\left(x_{0}, M\left(t_{0}\right)\right)<\delta$ the inequality $\rho(x(t), M(t))<\varepsilon, t \geq t_{0}$, holds. Reasoning as well as proof of Theorem 4 , brings us to inequalities

$$
\int_{\nu\left(\tau_{i}+0\right)}^{\nu\left(\tau_{i+1}+0\right)} \frac{\mathrm{d} s}{\varphi(s)} \leq-\gamma_{i+1}, \quad i=j+1, j+2, \ldots
$$

which implies that sequence $\left\{v\left(\tau_{j+k}+0\right)\right\}, k=1,2, \ldots$, is nonincreasing. Let $\beta=$ $\lim _{k \rightarrow \infty} v\left(\tau_{j+k}+0\right)$. Assume that $\beta>0$. Under such assumption from (3.18) we obtain:

$$
v\left(\tau_{j+n}+0\right) \leq v\left(\tau_{j+1}+0\right)-c \sum_{i=j+2}^{n} \gamma_{i}
$$

where $c=\min _{\beta \leq s \leq a(\epsilon)} \varphi(s)$. Since $v\left(\tau_{j+1}+0\right)-c \sum_{i=j+2}^{n} \gamma_{i} \rightarrow-\infty$ as $n \rightarrow \infty$, then inequality (3.19) is contradictory. The reason for the contradiction obtained is the assumption that $\beta>0$. So, $\beta=0$. In view of conditions of the theorem $(v(t)$, decreases on each interval of continuousity $\left(\tau_{i}, \tau_{i+1}\right], i=j+1, j+2, \ldots$, so $v(t) \rightarrow 0$ as $t \rightarrow \infty$, and, simultaneously, $\left.\lim _{t \rightarrow \infty} \rho(x(t)), M(t)\right)=0$. The theorem is proved.

Theorem 11. Let for system (3.1) there exist a continuously differentiable function $V(t, x)$ satisfying in domain $Z$ conditions $(3.2),(3.3),(3.10),(3.11)$.

If the sequence $\left\{\tau_{i}\right\}, i=1,2, \ldots$ satisfies inequality (3.9) and if there exist such positive numbers $a_{0}$ and $\gamma$ that for all $a \in\left(0, a_{0}\right]$

$$
\int_{a}^{\psi(a)} \frac{\mathrm{d} s}{\varphi(s)} \leq \theta-\gamma
$$

then the set $M$ is asymptotically stable with respect to system (3.1). Theorem 11 is a corollary of Theorem 10 .

Theorem 12. Let for system (3.1) there exist a continuously differentiable function $V(t, x)$ satisfying in domain $Z$ conditions (3.2), (3.3), (3.12), (3.13).

If there exists a positive number $a_{0}$ such that for all $a \in\left(0, a_{0}\right]$ for some $\gamma_{i}>$ $0, i=1,2, \ldots$, inequalities

$$
\int_{\psi_{i}(a)}^{a} \frac{\mathrm{d} s}{\varphi(s)} \geq \int_{\tau_{i-1}}^{\tau_{i}} \alpha(t) \mathrm{d} t+\gamma_{i}, \quad i=1,2, \ldots
$$

hold, where $\sum_{i=1}^{\infty} \gamma_{i}=\infty$, then the set $M$ is asymptotically stable with respect to system (3.1).

The proof of Theorem 12 resembles the proof of Theorem 10. 
Theorem 13. Let for system (3.1) there exist a continuously differentiable function $V(t, x)$ satisfying in domain $Z$ conditions (3.2), (3.3), (3.16), (3.17). If sequence $\left\{\tau_{i}\right\}, i=1,2, \ldots$, satisfies (3.15) and there exist positive numbers $a_{0}$ and $\gamma$ such that for arbitrary $a \in\left(0, a_{0}\right]$ inequality

$$
\int_{\psi(a)}^{a} \frac{\mathrm{d} s}{\varphi(s)} \geq \theta_{1}+\gamma
$$

holds, then the set $M$ is asymptotically stable with respect to system (3.1).

The assertion of Theorem 13 follows from Theorem 12.

Acknowledgement: The first author was supported in part by the Hungarian-Ukrainian Bilateral Science and Technology Cooperation Program through grant No. UK-3/99.

\section{REFERENCES}

[1] Samollenko, A.M. and Perestyuk, N.A.: Impulsive Differential Equations, Vishcha Shkola, Kiev, 1987, p. 28. (in Russian).

[2] Samoilenko, A.M. and Perestyuk, N.A.: Impulsive Differential Equations, Singapore etc.: World Scientific, 1995. - p. 462.

[3] Aknmetov, M.U. and Perestyuk, N.A.: On comparison method for differential systems with impulse effect, Differential Equations, 26(9), (1990), 1475-1483.

[4] Yoshizawa T.: Stability of sets and perturbed system, Funkcialaj Ekvacioj, 5, (1962), 31-69.

[5] Samollenko, A.M.: Study of dynamical systems by means of sign constant functions, Ukrainskii Matematicheskii Zhurnal, 24(3), (1972), 374-384.

[6] Zubov, V.I.: Stability of Motion, Moscow, Visshaya Shkola, 1973, p. 271.

[7] Bulgakov, N.G.: On the stability of invariant sets, Differential Equations, 18(2), (1982), 187-194.

[8] Ignatev, A.O.: Application Lyapunov direct method for the research of integral sets, Ukrainskii Matematicheskii Zhurnal, 44(10), (1992), 1342-1348.

[9] Gurgula, S.I. and Perestyuk, N.A.: On the second Lyapunov method in impulsive systems, Dokl. Acad. Nauk Ukrain. SSR, Ser.A.(10), (1982), 11-14.

[10] Gurgula, S.I. and Perestyuk, N.A.: On stability of solutions of impulsive systems, Vestnik Kievskogo Universiteta - Matematika i Mechanika, 23, (1981), 33-40.

[11] Gurgula, S.I.: A study of stability of solutions of impulsive systems by second Lyapunov method, Ukrainskii Matematicheskii Zhurnal, 34(1), (1982), 100-103.

[12] Kulev, G.K. and BAinov, D.D.: Global stability of sets for impulsive differential systems by Lyapunov's direct method, Comp. Math. Applic. 19(2), (1990), 17-28.

[13] LIU X.Z.: Stability results for impulsive differential systems with applications to population growth models, Dynamics and Stability of Systems, 9(2), (1994), 163-174.

[14] Perestyuk, N.A. and Chernikova, O.S.: Stability of solution of impulsive systems, Ukrainskii Matematicheskii Zhurnal, 49(1), (1997), 98-111.

[15] Perestyuk, N.A. and Chernikova, O.S.: On stability of invariant sets of discontinuous dynamical systems, Ukrainskii Matematicheskii Zhurnal, 53(1), (2001), 78-84. 